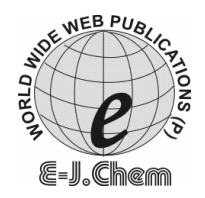

http://www.e-journals.net
ISSN: 0973-4945; CODEN ECJHAO

E-Journal of Chemistry

2009, 6(1), 53-60

\title{
High Performance Liquid Chromatography Method for Determination of Trace Amount of Ibutilide Fumarate in Pharmaceutical Manufacturing Environments
}

\author{
M. SATYA BABU*, PRATHAMA S. MAINKAR and Y.ANJANEYULU
}

Sai Advantium Pharma Limited (SAP Limited),

LB Nagar, Hyderabad,

Andhra Pradesh, India.

satya.babu7@gmail.com

Received 3 June 2008; Accepted 14 July 2008

\begin{abstract}
A rapid and sensitive RP-HPLC method with UV detection at $227 \mathrm{~nm}$ for routine analysis of washed MLs (mother liquors) from equipment after manufacturing and thereby cleaning of ibutilide fumarate active pharmaceutical ingredient was developed. Chromatography was performed with mobile phase containing a mixture of aqueous $0.01 \mathrm{M}$ potassium dihydrogen phosphate $\left(\mathrm{KH}_{2} \mathrm{PO}_{4}\right)$ and acetonitrile. The gradient elution was developed for better and optimized results. The developed method was validated for precision which includes system precision and method precision, accuracy, intra-day and using different system and finally linearity studies from 0.4 to $150 \%$. The method is ascertained to be having good repeatability and reproducibility. The $\%$ RSD for method precision ( 5 different preparations at test concentration) was observed to be 1.36 , wherein the system precision (6 repeated injections of same preparation) was observed to be 0.33 . The $\%$ recovery from 'Accuracy' studies yielded the recovery of $99-100 \%$ which indicates the capability of the method. The linearity of the method was studied right from 0.4 to $150 \%$ which shows the method is quite linear with a correlation coefficient of 0.9998 . The proposed method was simple, highly sensitive, precise, and accurate.The retention time less than $15 \mathrm{~min}$, indicated that the method is useful for routine testing of washed MLs from equipment after manufacturing and cleaning of ibutilide fumarate.
\end{abstract}

Keywords: Ibutilide fumarate, HPLC, Correlation coefficient, \%RSD, Active Pharmaceutical Ingredient, US FDA, 


\section{Introduction}

Ibutilide fumarate is an antiarrhythmic drug with predominantly class III (cardiac action potential prolongation) properties according to the Vaughan Williams Classification. The drug is available in the market as injection wherein each milliliter of injection contains 0.1 $\mathrm{mg}$ of ibutilide fumarate (equivalent to $0.087 \mathrm{mg}$ ibutilide free base), $0.189 \mathrm{mg}$ sodium acetate trihydrate, $8.90 \mathrm{mg}$ sodium chloride, hydrochloric acid to adjust $\mathrm{pH}$ to approximately 4.6 and water for injection. The ibutilide injections are available with the brand name of "COVERT" manufactured and distributed by Pharmacia Upjohn.

The chemical name for ibutilide fumarate is methanesulfonamide, $\mathrm{N}-\{4-\{4$-(ethylheptyl amino)-1 -hydroxybutyl $\}$ phenyl $\},(+)(-),(E)-2$-butenedioate (1:0.5) (hemifumarate salt). Its molecular formula is $\mathrm{C}_{22} \mathrm{H}_{38} \mathrm{~N}_{2} \mathrm{O}_{5} \mathrm{~S}$, and its molecular weight is 442.62 . Ibutilide fumarate is a white to off-white powder with an aqueous solubility of over $100 \mathrm{mg} / \mathrm{mL}$ at $\mathrm{pH} 7$ or lower.

The structural formula is depicted below:

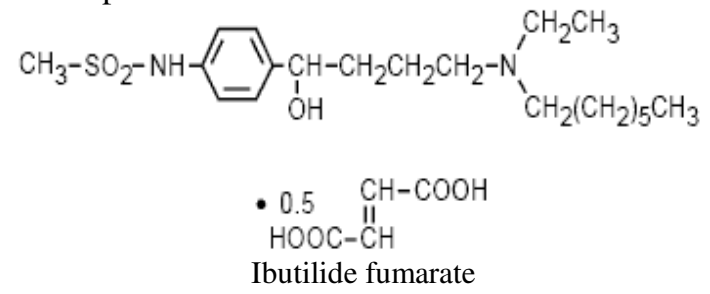

In the pharmaceutical manufacturing environment, cleaning of equipment plays a vital role where the assurance of carry over of previous product has to be within the regulatory acceptable limits. i.e., calculation of maximum allowable carryover (MAC) and there by applying the MAC to calculate allowable limit for rinse and swab sampling etc. The drug regulatory agencies, for example, US FDA, are very keen and particular about the capability of analytical method in terms of sensitivity, limit of detection, accuracy and precision.

So far, only the determination of ibutilide from human plasma using LC-tandem mass spectrometry ${ }^{1}$ and assaying of enantiomers of ibutilide and artilide using solid-phase extraction, derivatization and achiral-chiral column switching high performance liquid Chromatography ${ }^{2}$ have been described in literature. However, no procedure has been described for the determination of API at low levels (at ppm level) accurately and within the linear range. Hence, it was felt necessary to develop a liquid chromatographic procedure which would serve as a rapid and reliable method for determination of ibutilide fumarate at trace levels (even at $1 \mathrm{ppm}$ ).

In this work we have developed a selective and sensitive RP-High Performance Liquid Chromatography method which can conveniently detect the lowest possible amount of $0.001 \%$ of ibutilide in the equipment cleaning MLs with a good sensitivity, selectivity, repeatability and reproducibility.

\section{Experimental}

\section{Materials and reagents}

Ibutilide fumarate API was synthesized by a well known API/CRO company using the generic process developed and established by the same firm. The HPLC purity of the selected test sample is higher than the $99.9 \%$. A laboratory reagent grade Potassium dihydrogen phosphate was procured from 'Qualigens' for which the reported assay is $99.5 \%$. Additionally, the laboratory grade acetonitrile used in this experimentation was obtained from 'Merck' for which the purity is reported to be $99.8 \%$ 


\section{Chromatographic conditions and Instrumentation}

The RP-High Performance Liquid Chromatography was carried out on 'Waters' Liquid Chromatograph model 2695 equipped with quaternary pump, Photo Diode Array detector (PDA, waters \# 2996), column oven and auto sampler. The output signal was monitored and integrated using 'Empower 2' software.

Symmetry $\mathrm{C}_{8}$ column with $250 \mathrm{~mm}$ length and $4.6 \mathrm{~mm}$ dia with $5 \mu$ particle size has been optimized to obtain the best possible results. The other columns tried for development of this method are, Inertsil $\mathrm{C}_{8} 250 \times 4.6 \mathrm{~mm}, 5 \mu$ and $\mathrm{YMC} \mathrm{C}_{8} 250 \times 4.6 \mathrm{~mm}, 5 \mu$ (Figure 3). A mixture of $0.01 \mathrm{M}$ potassium dihydrogen phosphate $\left(\mathrm{KH}_{2} \mathrm{PO}_{4}\right)$ in water and acetonitrile was used as mobile phase. The gradient elution program has been developed and optimized for better, accurate and consistent results. The gradient program developed for this method is depicted below

\begin{tabular}{cc}
\hline Time & Solvent B \% (Acetonitrile) \\
\hline 0 & 25 \\
5 & 25 \\
15 & 90 \\
20 & 90 \\
25 & 25 \\
30 & 25 \\
\hline
\end{tabular}

The optimized flow rate was $1.0 \mathrm{~mL} / \mathrm{min}$ and the detection was at $227 \mathrm{~nm}$. The UV absorbance spectrum is depicted in Figure 1.

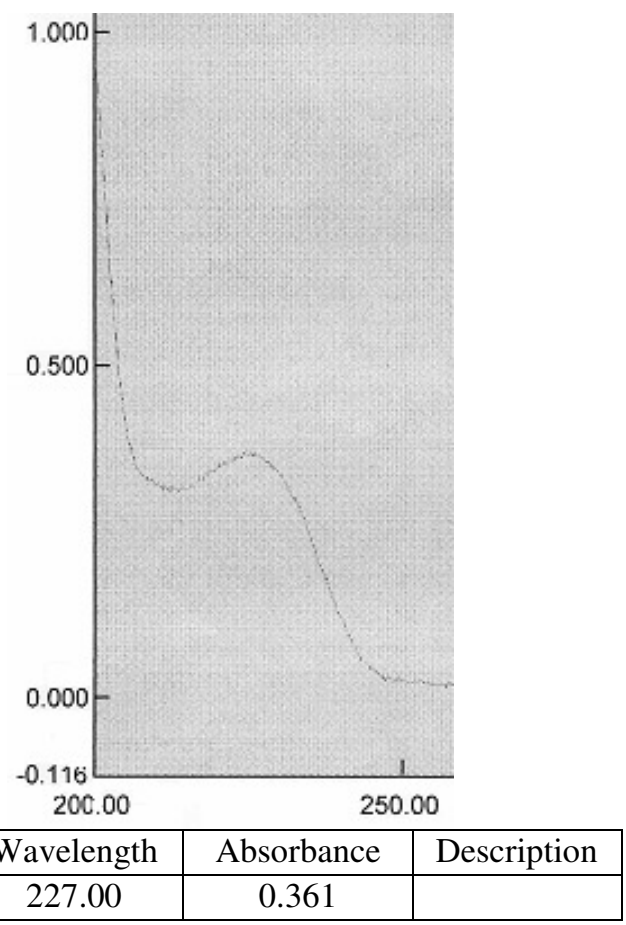

Figure 1. UV absorbance spectrum for ibutilide fumarate. 
The column temperature for these experiments was maintained at $40^{\circ} \mathrm{C}$. The test concentration of $0.25 \mathrm{mg} / \mathrm{mL}$ has been developed and optimized. The theoretical plates were observed to be 63528 where as the tailing factor is 1.53 (Table 1). The selectivity of Ibutilide has been shown in Figure 2.

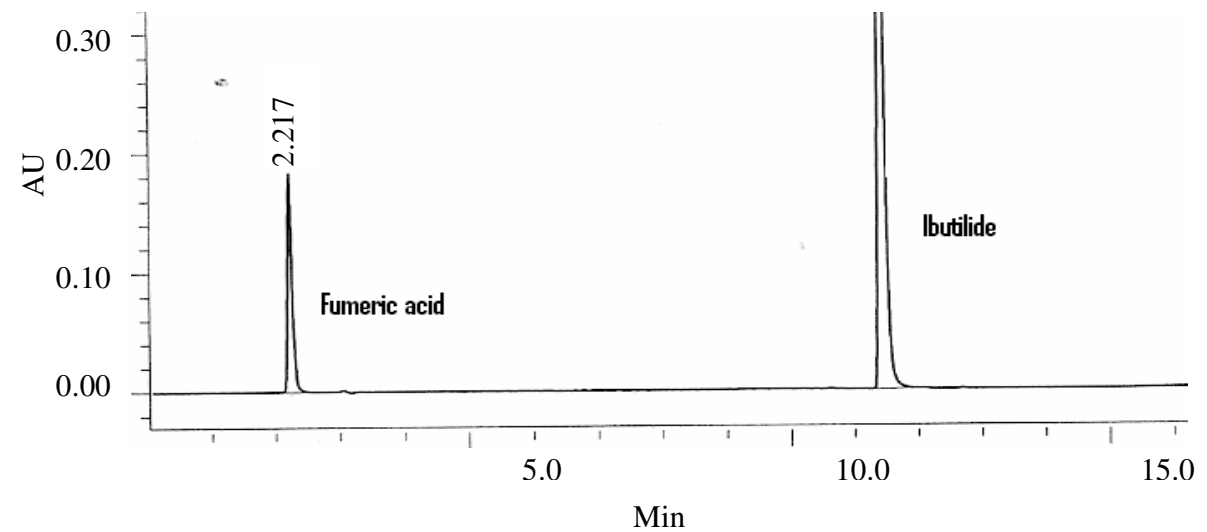

Figure 2. Typical chromatogram.

Table 1. System suitability report (symmetry C8 column, $250 \times 4.6 \mathrm{~mm}, 5 \mu$ )

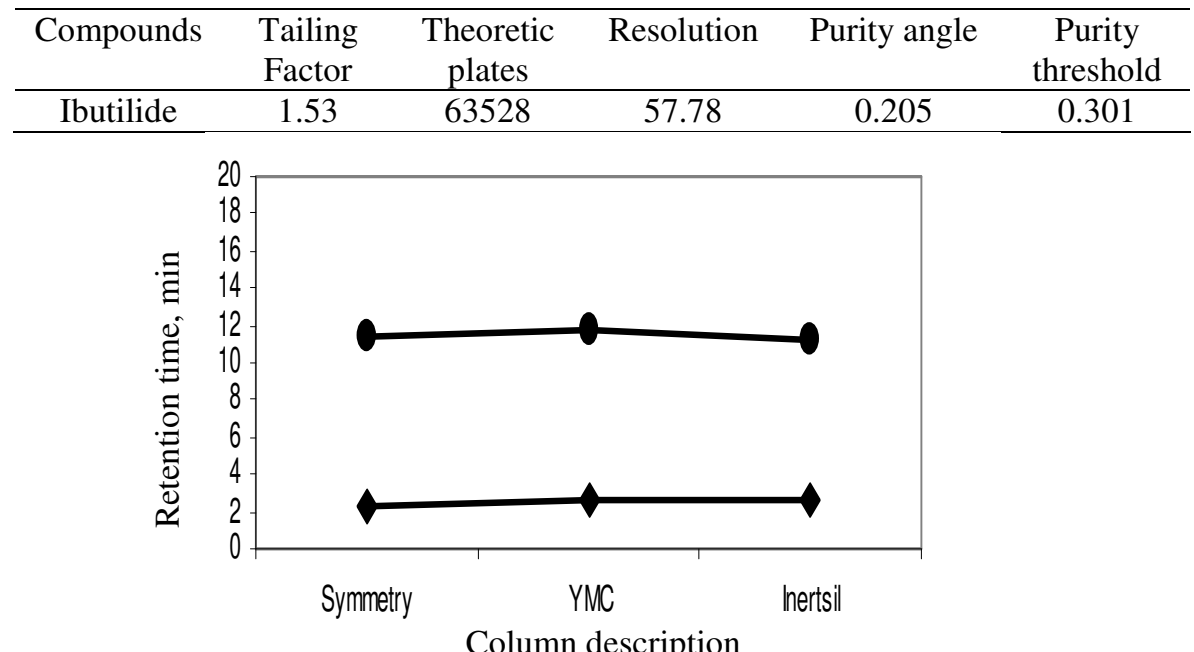

Figure 3. Retention behavior of ibutilide fumarate on various columns

The developed method has been meticulously studied to ensure the method precision, system precision, \% recovery (accuracy) and linearity. The method precision was studied by preparing the 5 different preparations of test concentration (i.e., $0.25 \mathrm{mg} / \mathrm{mL}$ ) and by injecting into the liquid chromatograph using the established method conditions. The area $\%$ for ibutilide fumarate obtained from these 5 runs was subjected to calculation of $\%$ relative standard deviation (\%RSD) which is observed to be 1.36. Similarly, the system precision is also studied prior to executing the accuracy and linearity studies by injecting the standard preparation for 6 different times to ensure the repeatability. The $\%$ area of for ibutilide obtained from these runs is subjected calculation of $\%$ RSD and observed to 0.33 . The above results ascertain that the method quite good and well reproducible and repeatable. 
The accuracy or $\%$ recovery studies were carried out at the concentrations of 150,100 and $50 \%$. The results obtained from these studies are quite encouraging and well within the standard norms. i.e., between 80 and $120 \%$. At $150 \%$ concentration, $98.8 \%$ recovery has been obtained. Similarly, at $100 \%$, the reported \% recovery was $100 \%$ and finally at $50 \%$ concentration, $100.33 \%$ of recovery has been reported. The correlation coefficient across the three concentrations \% recovery is plotted and observed to be 0.9997 .

The linearity of the method was established across 7 different concentrations starting from 0.4 to $150 \%$. The concentrations examined during this study are $0.4,25,50,75,100$, 125 and $150 \%$. The response obtained for these runs has been plotted and subjected to the calculation of correlation coefficient. The calculated correlation coefficient was 0.9998 where in the slope is 39088.47 and y-intercept was 46910.59 . The 0.9998 correlation coefficient indicates the method capability across the linear range.

\section{Results and Discussion}

The method precision as explained in the earlier section has been studied and results obtained for five different preparations are tabulated (Table 2) along with calculation for mean, standard deviation and \% relative standard deviation.

Table 2.

\begin{tabular}{ccc}
\hline Run & \% Area for ibutilide \\
\hline 1 & 4254499 & \\
2 & 4178681 & \\
3 & 4204323 & \\
4 & 4136631 & \\
5 & 4109116 & \\
\hline Mean & - & 4176650 \\
Standard Deviation & - & 56992.9 \\
\%RSD & - & 1.36
\end{tabular}

The system precision was studied by preparing the standard solution at test concentration i.e., $0.25 \mathrm{mg} / \mathrm{mL}$ and injected repeatedly for 6 times. The obtained results are shown in Table 3.

Table 3.

\begin{tabular}{ccc}
\hline Run \# & \% Area for ibutilide \\
\hline 1 & 4067956 & \\
2 & 4071621 & \\
3 & 4057677 & \\
4 & 4053835 & \\
5 & 4034411 & \\
6 & 4050562 & \\
\hline Mean & - & 4056010 \\
Standard Deviation & - & 13342.54 \\
\%RSD & - & 0.33
\end{tabular}

\section{Accuracy (\%recovery)}

The $\%$ recovery has been studied and established within the range of 50 to $150 \%$. The spiking study for each concentration along with amount recovered and thereby calculation of $\%$ recovery is tabulated in Table 4. 
Table 4.

\begin{tabular}{cccc}
\hline Description & $150 \%$ & $100 \%$ & $50 \%$ \\
\hline Wt of sample taken & $37.12 \mathrm{mg}$ & $25.02 \mathrm{mg}$ & $12.28 \mathrm{mg}$ \\
Run 1 (area) & 6181769 & 4244748 & 2085212 \\
Run 2 (area) & 6187764 & 4208639 & 2071999 \\
Run 3 (area) & 6257371 & 4255503 & 2101303 \\
Mean area & 6208968 & 4236297 & 2086171 \\
St. deviation & 42025.3 & 24548.5 & 14675.5 \\
\%RSD & 0.68 & 0.58 & 0.70 \\
Amount Recovered & $36.6708 \mathrm{mg}$ & $25.0200 \mathrm{mg}$ & $12.3211 \mathrm{mg}$ \\
\% Recovery & $98.8 \%$ & $100.0 \%$ & $100.3 \%$ \\
\hline
\end{tabular}

The response obtained for the various concentrations is plotted and observed to be linear (correlation coefficient -0.9997 ). The graphical representation of accuracy studies is depicted in Figure 4

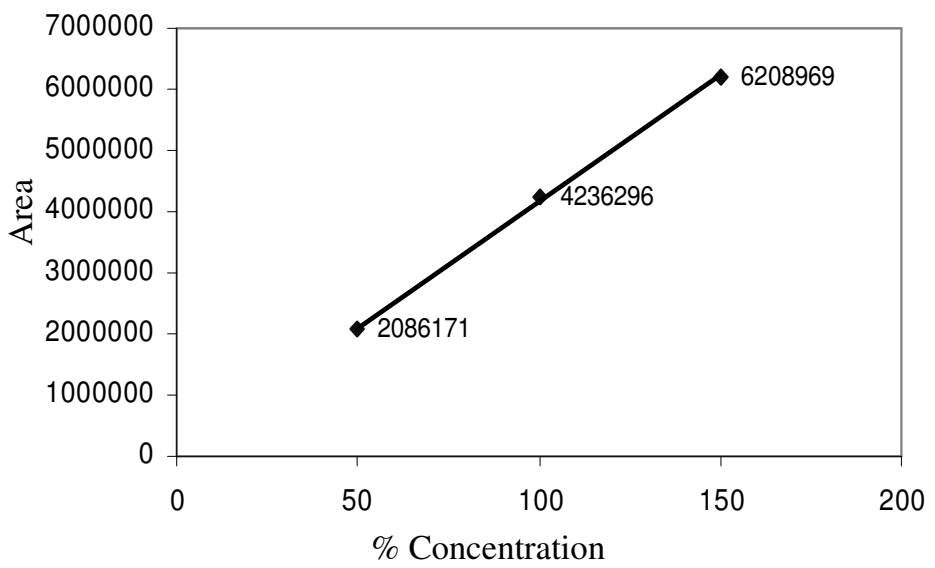

Figure 4. Ibutilide fumarate accuracy studies linearity plot

\begin{tabular}{cc}
\hline$\%$ Conc & Area \\
\hline 150 & 6208968 \\
100 & 4236296 \\
50 & 2086171
\end{tabular}

\section{Linearity}

Correlation Coefficient 0.9997

The linearity of the method was studied at seven concentrations including the test concentration. i.e., 150,125, 100, 75, 50, 25, 0.4\%. The obtained response was linear which apparently indicates the capability of method to reproduce/repeat the results within the linear range, consistently meeting the standard norms for method validation (ICH guidelines on validation analytical methods, Q2B). The results obtained for various experiments are depicted in Table 5. 
Table 5.

\begin{tabular}{cccccccc}
\hline Description & $150 \%$ & $125 \%$ & $100 \%$ & $75 \%$ & $50 \%$ & $25 \%$ & $\begin{array}{c}0.4 \%, 10 \\
\text { ppm }\end{array}$ \\
\hline Run 1 (area) & 5868003 & 4908454 & 3975013 & 3002320 & 2009878 & 1033565 & 16634 \\
Run 2 (area) & 5897040 & 4902367 & 3968418 & 3038337 & 2030476 & 1027204 & 16311 \\
Run 3 (area) & 5899360 & 4907563 & 3971339 & 3042297 & 2042955 & 1022749 & 16094 \\
Mean area & 5888134 & 4906128 & 3971590 & 3027651 & 2027770 & 1027839 & 16346 \\
Std & 17472.8 & 3287.5 & 3304.6 & 22026.8 & 16703.7 & 5435.9 & 271.7 \\
\% . & 0.2967 & 0.0670 & 0.0832 & 0.7275 & 0.8237 & 0.5289 & 1.6623 \\
\hline
\end{tabular}

The response obtained for each of the concentration is plotted by taking \% concentration on $x$-axis and area on $y$-axis. The results are then subjected to calculation of correlation coefficient after calculation of slope and $y$-intercept. The graphical representation for linearity studies is depicted in Figure 5.

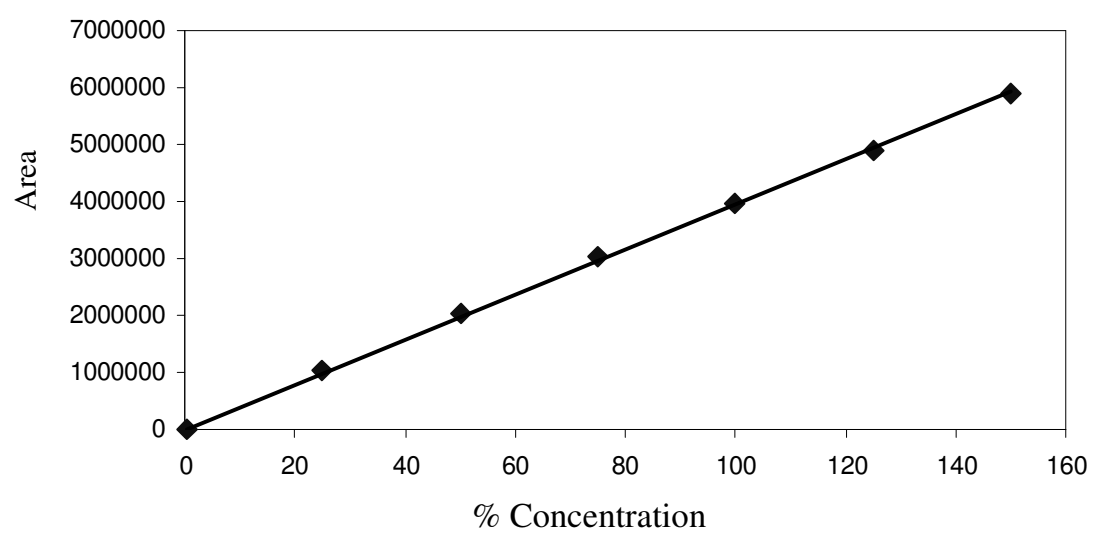

Figure 5. Ibutilide fumarate linearity studies plot

\begin{tabular}{cc}
\hline$\%$ Conc. & Area \\
\hline 150 & 5888134 \\
125 & 4906128 \\
100 & 3971590 \\
75 & 3027651 \\
50 & 2027770 \\
25 & 1027839 \\
0.4 & 16346 \\
\hline \multicolumn{3}{c}{ Slope - 39088 } \\
y-intercept - 46910 \\
Correlation coefficient 0.9998
\end{tabular}

\section{Conclusions}

The results obtained from these studies are well fit into the standard specifications stipulated by the regulatory agencies. The method is able to reproduce the results consistently even at $10 \mathrm{ppm}$ level and hence this can be conveniently used in the pharmaceutical manufacturing environment for equipment cleaning certification. 


\section{References}

1 Lei Tian, Yishi Li, Yiling Huand, Fuhua Kuang, Feiou Li and Li Hua. $J$ Chromatogr., 1998, 816(1-2), 81-85.

2 HSU C.-Y.L; Walgters R.R J Chromatogr., B, 1995, 667, 115-128.

3 Rodehamel J, "Cleaning and Maintenance," University of Wisconsin's Control Procedures in Drug Production Seminar, 1966, July 17-22, pp 82-87 William Blockstein, Editor, Published by the University of Wisconsin, L.O.C.\#66-64234

$4 \quad$ Harder S W, Pharm Technol., 1984, 8(5), 29-34.

$5 \quad$ Fourman G L and Mullen M V, Pharm Technol., 1993, 17(4), 54-60.

6. Chapter $<1225>$ Validation of Compendial Methods; US Pharmacopeia 23, United States Pharmacopeial Convention, Inc., Rockville MD: 1994

7 Compliance program guidance manual, Food and drug administration, Guide to inspection of validation of cleaning processes, July 1993.

8 International conference on Harmonization, (ICH) Q2B, Validation of Analytical Procedures, Methodology, May 1997. 


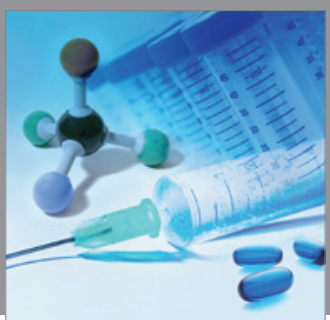

International Journal of

Medicinal Chemistry

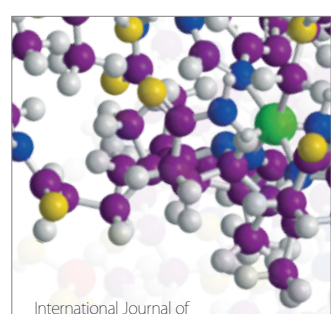

Carbohydrate Chemistry

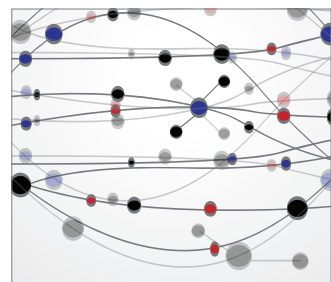

The Scientific World Journal
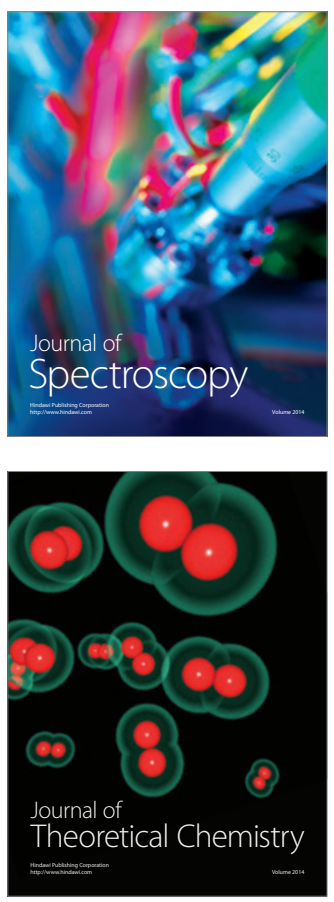
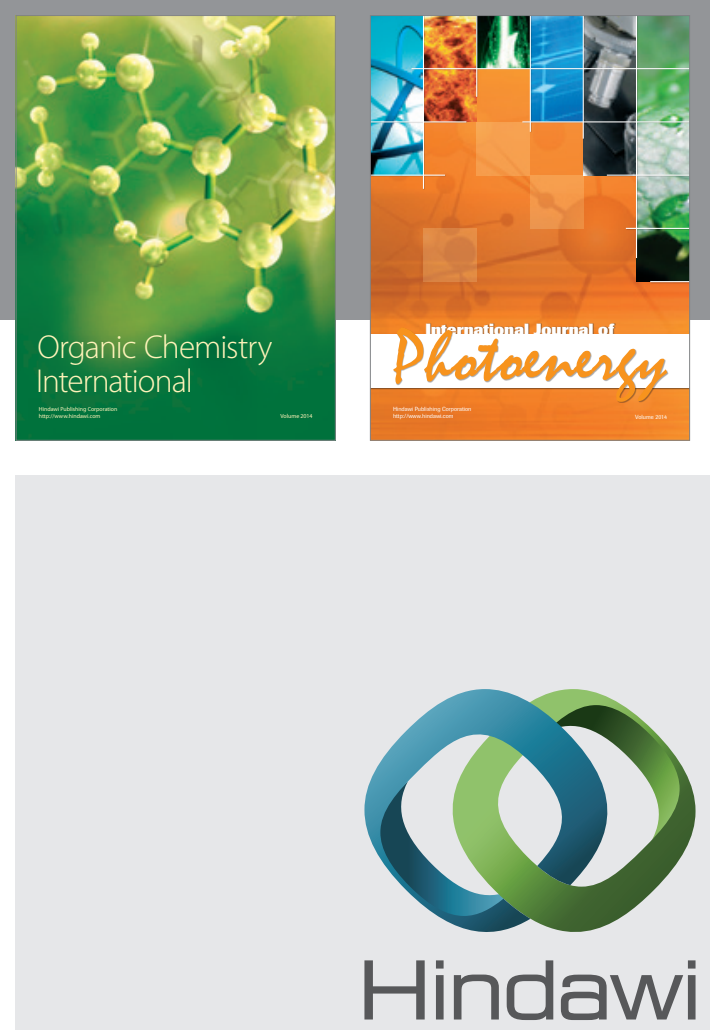

Submit your manuscripts at

http://www.hindawi.com
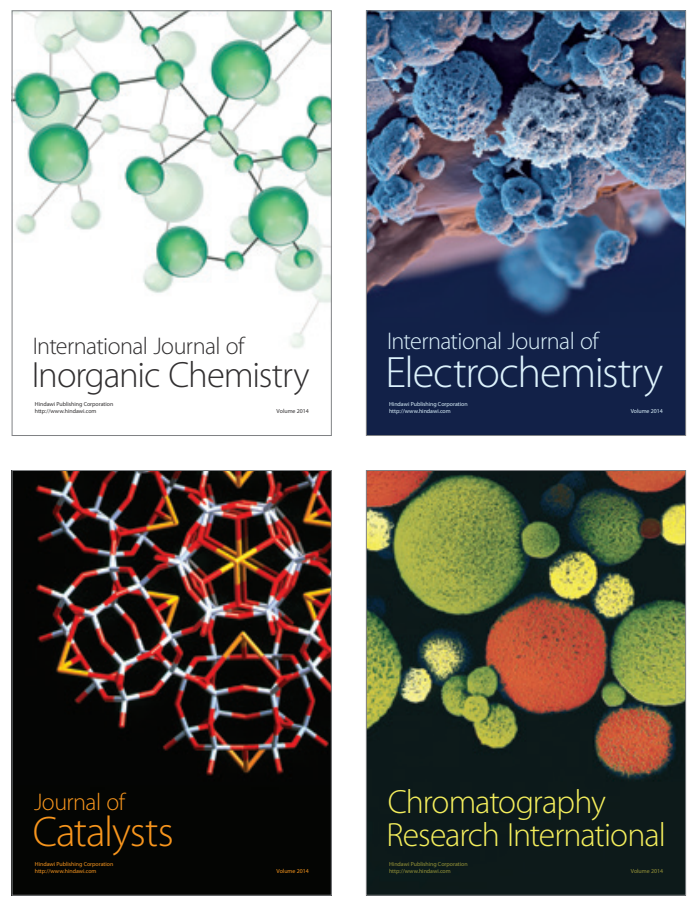
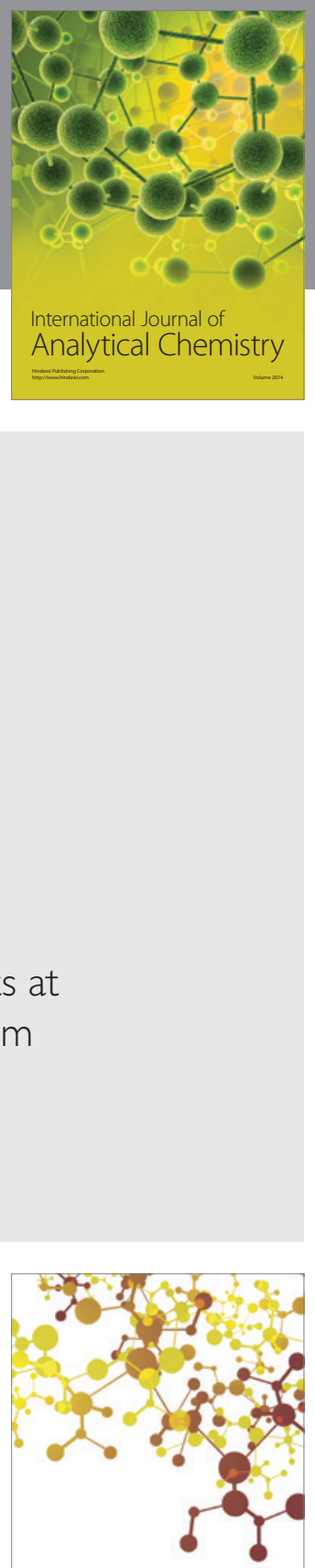

Journal of

Applied Chemistry
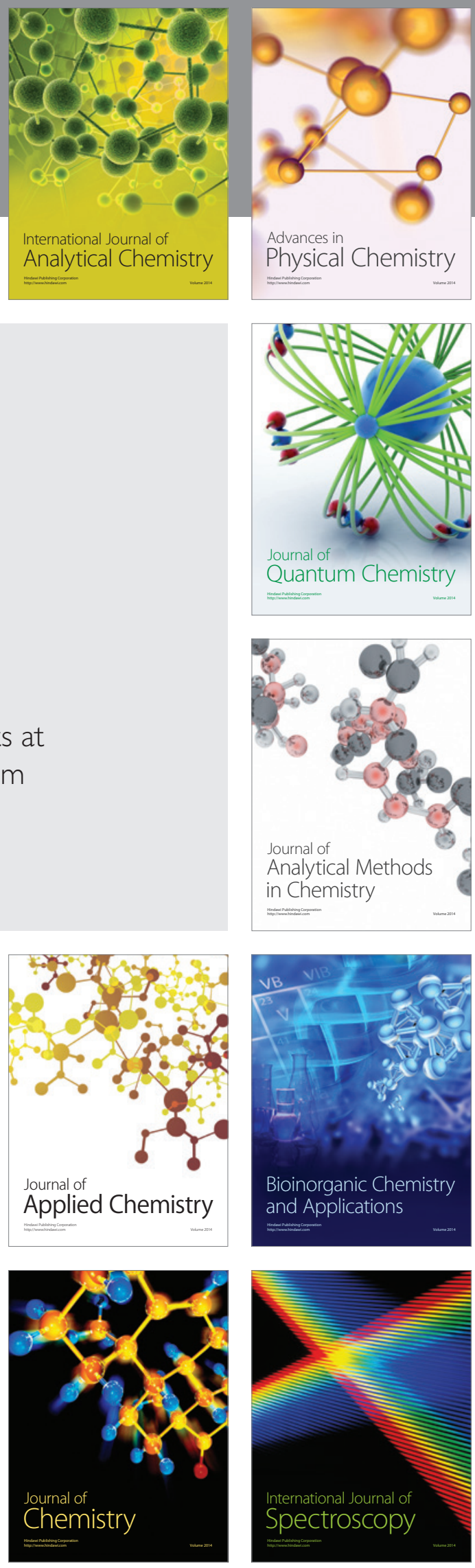